\title{
FIRST ORDER OUTAGE STATISTICS OF ASYMMETRICAL RF- OW DUAL-HOP RELAY COMMUNICATIONS
}

\author{
STEFAN PANIĆ ${ }^{*}$, SERGEI MOHANCHENKO ${ }^{2}$, ČASLAV STEFANOVIĆ ${ }^{1}$, MIHAJLO \\ STEFANOVIĆ
}

${ }^{1}$ Faculty of Natural Sciences and Mathematics, University of Priština, Kosovska Mitrovica, Serbia

${ }^{2}$ School of Computer Sciences and Robotics, National Research Tomsk Polytechnic University, Tomsk, Russia

${ }^{3}$ Faculty of Electronic Engineering, University of Niš, Niš, Serbia

\begin{abstract}
This paper addresses the first-order outage statistics of asymmetrical radio frequency (RF)-optical wireless (OW) relay systems over non turbulent-induced-fading (nTIF) and turbulent-induced-fading (TIF) channels. We rely on dual-hop amplify-and-forward relay (AFR) scheme and provide detailed mathematical development for derivation of novel exact analytical as well as novel closed form approximative expressions for: $i$ ). cumulative distribution function, $i$ i.) outage probability, and $i i i$.) average bit-error-rate. The system under consideration is modeled as the product of independent Nakagmi-m and double squared Nakagami-m (also known as Gamma-Gamma) random processes. The obtained results of the proposed system are graphically presented for RF -OW TIF and nTIF channel sets of parameters. Moreover, the detailed comparisons of exact and approximated numerical results whose derivation resorts on exponential Laplace approximation method (LAM) are provided and thoroughly examined for the considered RF-OW statistical measures.
\end{abstract}

Keywords: Gamma-Gamma, Laplace approximation, Nakagami-m, Outage statistics, RF-FSO relay systems.

\section{INTRODUCTION}

Optical wireless (OW) communicationsas well as asymmetrical radio frequency (RF)-OW communications are relevant research topic within academia and industry for future 5G and even beyond 5G (B5G) network deployments (Hamza et al., 2018; Khalighi \& Uysal, 2014; Illi et al., 2017; Douik et al., 2016). The OW communications are primarily intended to speed up the transmission rate and ensure higher capacity and wider bandwidth compared to RF links. Moreover, OW communications, especially free space optical (FSO) communications which operates at near infrared part of the spectrum are i.) cost effective, ii.) spectrum license free, iii.) channel interference free. On the other hand, one of the main FSO system performance impairments is the impact of atmospheric turbulence due to the small- and large-scales atmospheric cells. Weather conditions as well as misalignment of the system's transmitter-receiver apparatus can cause further degradation of the system performance stability.

The relay system techniques represent efficient way to speed up data-rate, extend coverage and save energy but also to efficiently merge different wireless technologies (Zedini et al., 2014; Anees \& Bhatnagar, 2015; Petkovic et al., 2017; Zedini et al., 2015; Stefanovic et al., 2019a). Moreover, amplify-andforward relay (AFR) scheme plays an important role in all-RF, RF-FSO and all-FSO relay systems (Stefanovic, 2017; Karimi \& Masoumeh, 2011; Petkovic \& Trpovski, 2018) and in some cases

* Corresponding author: stefan.panic@ @pr.ac.rs

MATHEMATICS, COMPUTER SCIENCE AND MECHANICS the signal envelope, signal-to-interference ratio (SIR) and signalto-noise ratio (SNR) can be modeled as the product of two or more random processes (RPs) (Zlatanov et al., 2008; Stefanovic et al., 2018; Milosevic et al., 2018; Stefanovic et al., 2019b; Talha \& Pätzold, 2007; Issaid \& Alouini, 2019).

Namely, Nakagami-m (Nm) RP can address RF links over non turbulence induced fading (nTIF) channels (Nakagami, 1960) while double squared Nakagami-m (d-sNm), also known as gamma-gamma RP can address FSO links over moderate to strong turbulence induced fading (TIF) channels (Andrews \& Phillips, 2005; Vetelino et al., 2007; Al-Ahmadi, 2014). The papers (Zedini et al., 2014; Anees \& Bhatnagar, 2015; Petkovic et al., 2017) address mixed RF-FSO relay systems over Nm nTIF channels and d-sNm TIF channels and provide closed form analytical results for the first order statistical measures expressed through Meijer's G function. In paper (Zedini et al., 2015), Nm nTIF and d-sNm TIF are used to address cooperative mixed RFFSO relay link and obtained analytical results are given in terms of H-fox and Meijer's G functions. Moreover, in (Stefanovic et al., 2019a) the closed form analytical expressions for second order statistics of the products of Nm, d-sNm and Nm RPs are derived by Laplace approximation method (LAM) and efficiently applied to address TIF and nTIF channels of mixed triple-hop RF-FSO-RF vehicle-to-vehicle (V2V) AFR communications.

It is important to note that LAM already plays an important role in performance analysis of wireless communicationsystems (Stefanovic et al., 2019a; Stefanovic, 2017; Zlatanov et al., 2008; Stefanovic et al., 2018; Milosevic et al., 2018; Stefanovic et al., 2019b; Hajri et al., 2018). Moreover, LAM can provide precise 
approximations by solving complex many-folded integrals and significantly decrease computational time of complex analytical expressions. The proofs that LAM is able to provide precise results even under sub-asymptotic conditions is provided in (Butler \& Wood, 2002). Another remark of the LAM is its generality and simplicity of application, which in many cases can provide fast computing closed form accurate approximations derived from latent Gaussian models (Wang, 2010).

In this paper we provide comprehensive mathematical development for computing novel exact expressions as well as novel closed form approximative expressions for $i$.) cumulative distribution function, ii.) outage probability, iii.) and average bit error rate of the product of $\mathrm{Nm}$ and d-sNm RPs. Moreover, we rely on exponential LAM for derivation of closed form analytical expressions for the first order statistical measures. The obtained results are further used to address asymmetrical RF-FSO dualhop AFR system in the case when nTIF (fading over RF channel) and TIF (turbulence induced fading over FSO channel) are the main cause of the system model performance degradation.

To the best of author's knowledge there is no paper in open technical literature that applies exponential LAM for derivation of the first order outage statistics of RF-FSO AFR system over Nm nTIF and d-sNm TIF channels.

\section{SYSTEM MODEL OF RF-FSO DUAL-HOP AFR COMMUNICATIONS}

We model the mixed RF-FSO dual-hop AFR system as the product of the independent $\mathrm{Nm}$ random process (RP), $y_{N m_{1}}$ and gamma-gamma (GG) RP, $y_{G G}$. Further, we express GG as double squared Nakagami-m (d-sNm) RP, $y_{G G}=y_{N m_{2}}^{2} y_{N m_{3}}^{2}$. Thus, we model the output signal envelope $x_{\text {out }}$ as the product of the independent RPs, $x_{\text {out }}=\underbrace{y_{N m_{1}}}_{R F} \underbrace{y_{G G}}_{F S O}=\underbrace{y_{N m_{1}}}_{R F} \underbrace{y_{N m_{2}}^{2} y_{N m_{3}}^{2}}_{F S O}$. where,

$$
p_{Y_{N m_{i}}}\left(y_{N m_{i}}\right)=\frac{2\left(m_{i} / \Omega_{i}\right)^{m_{i}}}{\Gamma\left(m_{i}\right)} y_{N m_{i}}^{2 m_{i}-1} e^{-\frac{m_{i} y_{N m_{i}}^{2}}{\Omega_{i}}}, i=1,3 .
$$

whose average powers and fading severity parameters of RF Nm and FSO d-sNm RPs are, respectively, $\Omega_{1}=\Omega_{R F}, m_{1}=m_{R F}$.

$$
\begin{aligned}
& \Omega_{2}=\Omega_{3}=1, \\
& m_{2}=\alpha=\left[\exp \left(\frac{0.49 \delta^{2}}{\left(1+0.18 d^{2}+0.56 \delta^{12 / 5}\right)^{7 / 6}}\right)-1\right]^{-1}, \\
& m_{3}=\beta=\left[\exp \left(\frac{0.51 \delta^{2}\left(1+0.69 \delta^{12 / 5}\right)^{-5 / 6}}{\left(1+0.9 d^{2}+0.62 d^{2} \delta^{12 / 5}\right)^{5 / 6}}\right)-1\right]^{-1} .
\end{aligned}
$$

where $\alpha$ and $\beta$ are small-scale and large-scale cells related to atmospheric conditions, respectively, $\delta^{2}=0.5 C_{n}^{2} k^{7 / 6} L^{11 / 6}$ is the
Rytov variance and $d=\sqrt{k D^{2} / 4 L}$ is the optical wave number. Further, $C_{n}^{2}$ is Refractive index, $k=2 \pi / \lambda$ is wave-number $(\lambda$ wavelength), $D$ is receiver aperture diameter and $L$ is propagation distance.

\section{FIRST ORDER STATISTICS OF RF-FSO DUAL-HOP AFR COMMUNICATIONS}

\section{Probability Density Function (PDF)}

The PDF of $x_{\text {out }}$ can be expressed through joint and conditional probabilities as:

$$
\begin{aligned}
p_{X_{\text {out }}}\left(x_{\text {out }}\right) & =\int_{0}^{\infty} d y_{N m_{2}} \int_{0}^{\infty}\left|\frac{d y_{N m_{1}}}{d x_{\text {out }}}\right| p_{Y_{N m_{1}}}\left(\frac{x_{\text {out }}}{y_{N m_{2}}^{2} y_{N m_{3}}^{2}}\right) \\
& \times p_{Y_{N m_{2}}}\left(y_{N m_{2}}\right) p_{Y_{N m_{3}}}\left(y_{N m_{3}}\right) d y_{N m_{3}} .
\end{aligned}
$$

\section{Cumulative Distribution Function (CDF)}

The CDF of $x_{\text {out }}$ can be calculated by using (Simon \& Alouini, 2000; Gradshteyn \& Ryzhik, 2000, Equation 3.381.1 and Equation 8.352.1), respectively for the case where $m_{R F}$ is positive integer,

$$
\begin{aligned}
F_{X_{\text {out }}}\left(x_{\text {out }}\right) & =\int_{0}^{x_{\text {out }}} p_{X_{\text {out }}}(t) d t=\frac{4 \alpha^{\alpha} \beta^{\beta}}{\Gamma\left(m_{R F}\right) \Gamma(\alpha) \Gamma(\beta)}\left(m_{R F}-1\right) ! \\
& \times\left(\frac{\Gamma(\alpha) \Gamma(\beta)}{4 \alpha^{\alpha} \beta^{\beta}}-\sum_{k=0}^{m_{R F}-1} \frac{\left(\frac{m_{R F} x_{\text {out }}^{2}}{\Omega_{R F}}\right)^{k}\left(\frac{\Omega_{R F}}{m_{R F}}\right)^{m_{R F}}}{k !} J_{1}\right)(6)
\end{aligned}
$$

where,

$$
\begin{aligned}
J_{1} & =\int_{0}^{\infty} d y_{N m_{2}} \\
& \times \int_{0}^{\infty} e^{-\alpha y_{N m_{2}}^{2}-\beta y_{N m_{3}}^{2}-\frac{m_{R F} x_{O u t}^{2}}{\Omega_{R F} y_{N m_{2}} y_{N m_{3}}^{2}}+(2 \alpha-4 k-1) \ln \left(y_{N m_{2}}\right)+(2 \beta-4 k-1) \ln \left(y_{N m_{3}}\right)} d y_{N m_{3}} .
\end{aligned}
$$

The integral $J_{1}$ in (6) can be solved by applying exponential LAM for two folded integrals (Zlatanov et al., 2008, Equation I.3).

$$
\begin{aligned}
& \int_{0}^{\infty} d y_{N m_{2}} \int_{0}^{\infty} d f_{1}\left(y_{N m_{2}}, y_{N m_{3}}\right) e^{-T f_{2}\left(y_{N m_{2}}, y_{N m_{3}}\right)} d y_{N m_{3}} \\
& \approx \frac{2 \pi}{T} \frac{f_{1}\left(y_{N m_{2}}, y_{N m_{3}}\right)}{\sqrt{\operatorname{det} H}} e^{-T f_{2}\left(y_{N m_{2}}, y_{N m_{3}}\right)} .
\end{aligned}
$$

It has been shown in (Butler \& Wood, 2002) that accurate results can be obtained for real value parameter, $T=1$. Further, the LAM, also known as exponential LAM for constant multivariate function $f_{1}$ and variable multivariate function $f_{2}$ is considered in 
(Harding \& Hausman, 2007). Accordingly, the arguments in Eq. 7 are, respectively, $T=1, f_{1}\left(y_{N m_{20}}, y_{N m_{30}}\right)=1$,

$$
\begin{aligned}
& f_{1}\left(y_{N m_{20}}, y_{N m_{30}}\right)=-\alpha y_{N m_{20}}^{2}-\beta y_{N m_{30}}^{2}-\frac{m_{R F} x_{\text {out }}^{2}}{\Omega_{R F} y_{N m_{20}}^{4} y_{N m_{30}}^{4}} \\
& +(2 \alpha-4 k-1) \ln \left(y_{N m_{20}}\right)+(2 \beta-4 k-1) \ln \left(y_{N m_{30}}\right)
\end{aligned}
$$

Further, the Hessian matrix $H$ in Eq. 7 is,

$$
H=\left|\begin{array}{ll}
\frac{\partial^{2} f_{2}\left(y_{N m_{20}}, y_{N m_{30}}\right)}{\partial y_{N m_{20}}^{2}} & \frac{\partial^{2} f_{2}\left(y_{N m_{20}}, y_{N m_{30}}\right)}{\partial y_{N m_{20}} \partial y_{N m_{30}}} \\
\frac{\partial^{2} f_{2}\left(y_{N m_{20}}, y_{N m_{30}}\right)}{\partial y_{N m_{30}} \partial y_{N m_{20}}} & \frac{\partial^{2} f_{2}\left(y_{N m_{20}}, y_{N m_{30}}\right)}{\partial y_{N m_{30}}^{2}}
\end{array}\right|,
$$

while, $y_{N m_{20}}$ and $y_{\mathrm{Nm}_{30}}$ are real and positive values obtained from the following equations,

$$
\begin{aligned}
& \frac{\partial f_{2}\left(y_{N m_{20}}, y_{N m_{30}}\right)}{\partial y_{N m_{20}}}=0, \\
& \frac{\partial f_{2}\left(y_{N m_{20}}, y_{N m_{30}}\right)}{\partial y_{N m_{30}}}=0 .
\end{aligned}
$$

\section{Outage Probability (Pout)}

The Pout of RF-FSO AFR proposed system is defined as the probability that the output signal goes below the outage threshold $x_{t h, R F-F S O}$ (Simon \& Alouini, 2000):

$$
P_{\text {out }}\left(x_{\text {th }, R F-F S O}\right)=\int_{0}^{x_{t h, R F-F S O}} p_{x_{\text {out }}}(t) d t=F_{x_{\text {out }}}\left(x_{t h, R F-F S O}\right) .
$$

\section{Average Bit Error Rate (BER)}

The average BER, $B E R_{R F-F S O}$ by definition for different binary modulations can be evaluated with (Anees \& Bhatnagar, 2015, Equation 23):

$$
B E R_{R F-F S O}=\frac{q^{p}}{2 \Gamma(p)} \int_{0}^{\infty} \exp \left(-q x_{\text {out }}\right) x_{\text {out }}^{p-1} F_{X_{\text {out }}}\left(x_{\text {out }}\right) d x_{\text {out }} .
$$

By substituting (5) in (Anees \& Bhatnagar, 2015, Equation 23), we obtain:

$$
\begin{aligned}
B E R_{R F-F S O} & =\frac{q^{p}}{2 \Gamma(p)} \frac{4 \alpha^{\alpha} \beta^{\beta}}{\Gamma\left(m_{R F}\right) \Gamma(\alpha) \Gamma(\beta)}\left(m_{R F}-1\right) ! \\
& \times\left(\frac{\Gamma(\alpha) \Gamma(\beta) \Gamma(p)}{4 \alpha^{\alpha} \beta^{\beta} q^{p}}-\sum_{k=0}^{m_{R F}-1} \frac{\left(m_{R F}\right)^{k}}{k !} J_{2}\right) .
\end{aligned}
$$

$$
\begin{aligned}
J_{2} & =\int_{0}^{\infty} d y_{N m_{2}} \int_{0}^{\infty} d y_{N m_{3}} \int_{0}^{\infty} e^{-\alpha y_{N m_{2}}^{2}-\beta y_{N m_{3}}^{2}-\frac{m_{R F} x_{\text {out }}^{2}}{\Omega_{R F} y_{N m_{2}}^{4} y_{N m_{3}}^{4}}} \\
& \times e^{q x_{\text {out }}(2 \alpha-4 k-1) \ln \left(y_{N m_{2}}\right)+(2 \beta-4 k-1) \ln \left(y_{N m_{3}}\right)+(p+2 k-1) \ln \left(x_{\text {out }}\right)} d x_{\text {out }} .
\end{aligned}
$$

where $p$ and $q$ denote parameters for different binary modulation such as:

- coherent binary frequency shift keying (CBFSK) for $p=0.5$ and $q=0.5$,

- coherent binary phase shift keying (CBPSK) for $p=0.5$ and $q=1$,

- non-coherent binary frequency shift keying (NBFSK) for $p=1, q=0.5$,

- differential binary phase shift keying (DBPSK) for $p=1$, $q=1$.

The closed form approximation for $B E R_{R F-F S O}$ can be obtained by evaluating $J_{2}$ in Eq. (13) by using exponential LAM for three folded integrals (Zlatanov et al., 2008, Equation I.3):

$$
\begin{aligned}
& \int_{0}^{\infty} d y_{N m_{2}} \int_{0}^{\infty} d y_{N m_{3}} \int_{0}^{\infty} d f_{1}\left(y_{N m_{2}}, y_{N m_{3}}, x_{\text {out }}\right) e^{-T f_{2}\left(y_{N m_{2}}, y_{N m_{3}}, x_{\text {out }}\right)} d x_{\text {out }} \\
& \approx \frac{2 \pi}{T} \frac{f_{1}\left(y_{N m_{20}}, y_{N m_{30}}, x_{\text {out }}\right)}{\sqrt{\operatorname{det} H}} e^{-T f_{2}\left(y_{N m_{20}}, y_{N m_{30}}, x_{\text {out }}\right)} .
\end{aligned}
$$

where the parameters in Eq. 15 are, respectively, $T=1$, $f_{1}\left(y_{N m_{2}}, y_{N m_{3}}, x_{\text {out }}\right)=1$,

$$
\begin{aligned}
& f_{2}\left(y_{N m_{20}}, y_{N m_{30}}, x_{\text {out }}\right)=-\alpha y_{N m_{20}}^{2}-\beta y_{N m_{30}}^{2}-\frac{m_{R F} x_{\text {out }}^{2}}{\Omega_{R F} y_{N m_{20}}^{4} y_{N m_{30}}^{4}} \\
& \quad-q x_{\text {out } 0}+(2 \alpha-4 k-1) \ln \left(y_{N m_{20}}\right)+(2 \beta-4 k-1) \ln \left(y_{N m_{30}}\right) \\
& +(p+2 k-1) \ln \left(x_{\text {out } 0}\right)
\end{aligned}
$$

Further, $y_{N m_{20}}, y_{N m_{30}}, x_{\text {out } 0}$ and $H$ in Eq. 14 can be evaluated from the following equations, respectively, $\frac{\partial^{2} f_{2}\left(y_{N m_{20}}, y_{N m_{30}}, x_{\text {out } 0}\right)}{\partial y_{N m_{20}}}=0, \quad \frac{\partial^{2} f_{2}\left(y_{N m_{20}}, y_{N m_{30}}, x_{\text {out } 0}\right)}{\partial y_{N m_{30}}}=0$, $\frac{\partial^{2} f_{2}\left(y_{N m_{20}}, y_{N m_{30}}, x_{\text {out } 0}\right)}{\partial x_{\text {out } 0}}=0$.

$H=\left|\begin{array}{llll}\frac{\partial^{2} f_{2}\left(y_{N m_{20}}, y_{N m_{30}}, x_{\text {out } 0}\right)}{\partial y_{N m_{20}}^{2}} & \frac{\partial^{2} f_{2}\left(y_{N m_{20}}, y_{N m_{30}}, x_{\text {out } 0}\right)}{\partial y_{N m_{20}} \partial y_{N m_{30}}} & \frac{\partial^{2} f_{2}\left(y_{N m_{20}}, y_{N m_{30}}, x_{\text {out } 0}\right)}{\partial y_{N m_{20}} \partial x_{\text {out } 0}} \\ \frac{\partial^{2} f_{2}\left(y_{N m_{20}}, y_{N m_{30}}, x_{\text {out } 0}\right)}{\partial y_{N m_{30}} \partial y_{N m_{20}}} & \frac{\partial^{2} f_{2}\left(y_{N m_{20}}, y_{N m_{30}}, x_{\text {out } 0}\right)}{\partial y_{N m_{30}}^{2}} & \frac{\partial^{2} f_{2}\left(y_{N m_{20}}, y_{N m_{30}}, x_{\text {out } 0}\right)}{\partial y_{N m_{30}} \partial x_{\text {out } 0}} \\ \frac{\partial^{2} f_{2}\left(y_{N m_{20}}, y_{N m_{30}}, x_{\text {out } 0}\right)}{\partial x_{\text {out } 0} \partial y_{N m_{20}}} & \frac{\partial^{2} f_{2}\left(y_{N m_{20}}, y_{N m_{30}}, x_{\text {out } 0}\right)}{\partial x_{\text {out } 0} \partial y_{N m_{30}}} & \frac{\partial^{2} f_{2}\left(y_{N m_{20}}, y_{N m_{30}}, x_{\text {out } 0}\right)}{\partial x_{\text {out }}^{2}}\end{array}\right|$,

where, 


\section{NUMERICAL RESULTS}

In numerical results we provide performance analysis as well as comparison of exact and approximated results of the first order outage statistics under weak, moderate and strong nTIF and TIF conditions of the RF-FSO dual-hop AFR system.

\section{First order Statistics}

The FSO section of dual-hop RF- FSO AFR relay link is modeled with d-sNmRP, where numerical results are evaluated for different optical fading severity parameters $(\alpha, \beta)$ and for different values of irradiance variance $\sigma_{d-s N_{m}^{2}}=\frac{1}{\alpha}+\frac{1}{\beta}+\frac{1}{\alpha \beta}$. The RF section of dual-hop RF-FSO AFR is modeled with $\mathrm{Nm}$ $\mathrm{RP}$ and evaluated for RF fading severity parameter $m_{R F}$.

\section{- Outage Probability}

The $P_{\text {out }}\left(x_{t h, R F-F S O}\right)$ for normalized $\Omega_{R F}=1$ under weak $\left(\alpha=4, \beta=4, m_{R F}=4\right)$, moderate to weak $\left(\alpha=3, \beta=3, m_{R F}=3\right)$, moderate to strong $\left(\alpha=2, \beta=2, m_{R F}=2\right)$ and strong $(\alpha=1, \beta=1$, $\left.m_{R F}=1\right)$ nTIF and TIF channel conditions is shown in Figure 1. It can be seen that $P_{\text {out }}\left(x_{t h, R F-F S O}\right)$ approximated by exponential LAM for the considered nTIF and TIF severity values fits well with exact analytical expression (Eq. 6) especially for higher $P_{\text {out }}\left(x_{t h, R F-F S O}\right) \mathrm{dB}$ values. It is evident that the system performance improvement can be achieved by increasing TIF and nTIF severity parameters since $P_{o u t}\left(x_{t h, R F-F S O}\right)$ decreases.

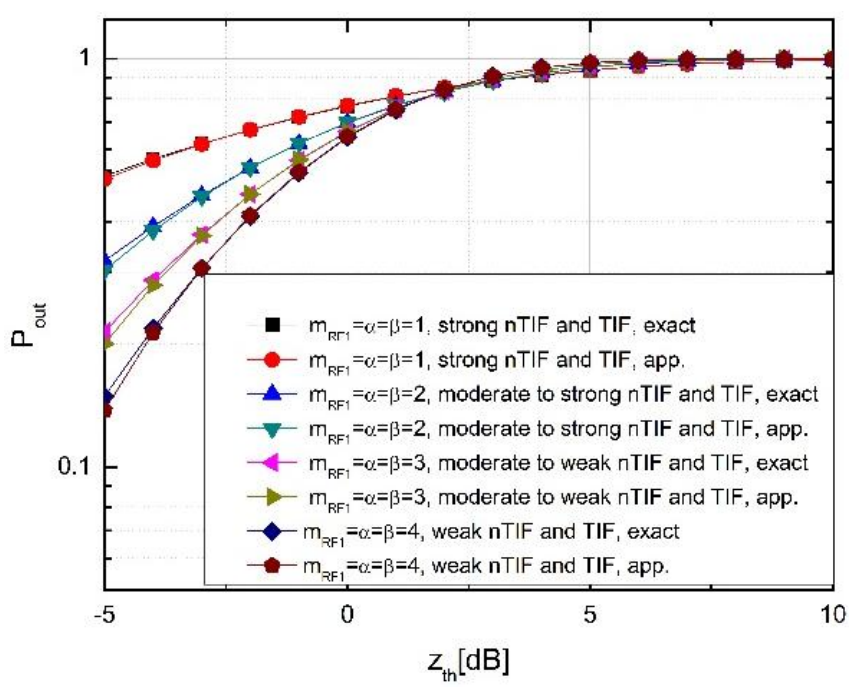

Figure 1. Comparison of exact and approximated results for $P_{\text {out }}$ under weak, moderate and strong TIF and nTIF channel conditions.

\section{- Average Bit Error Rate}

The average bit error rate versus $\Omega_{R F}$ for different binary modulation schemes under weak $\left(\alpha=3, \beta=3, m_{R F}=3\right)$, moderate $\left(\alpha=2, \beta=2, m_{R F}=2\right)$ and strong $\left(\alpha=1, \beta=1, m_{R F}=1\right) \mathrm{nTIF}$ and TIF channel conditions is presented in Figure 2. As expected, under moderate and weak TIF and nTIF channel conditions, BER noticeably decreases what can enable RF-FSO AFR system performance improvement.

Moreover, it can be seen that nTIF and TIF severity parameters $\left(\alpha, \beta, m_{R F}\right)$ have stronger impact on $B E R_{R F-F S O}$ than considered binary modulations. In most of the observed range and under considered nTIF and TIF channel conditions the best performance results relating to $B E R_{R F-F S O}$ can be achieved for CBPSK. The comparison of exact analytical expression and approximated closed form expression for $B E R_{R F-F S O}$ of DBPSK modulated signal are provided in Figure 3. It can be noticed that exponential LAM fails to match approximation with exact analytical results for higher $\Omega_{R F} \mathrm{~dB}$ values.

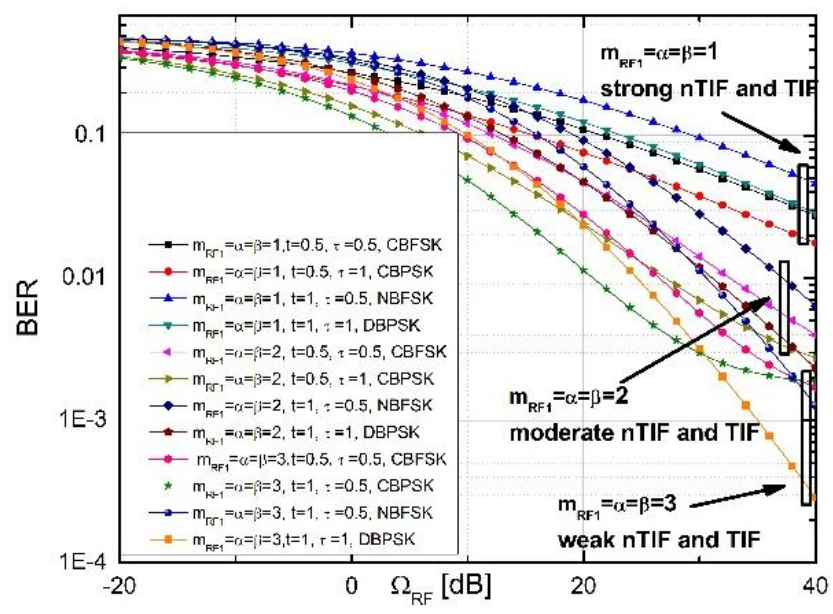

Figure 2. BER versus $\Omega_{R F}$ under weak, moderate and strong TIF and nTIF channel conditions and for different binary modulation schemes.

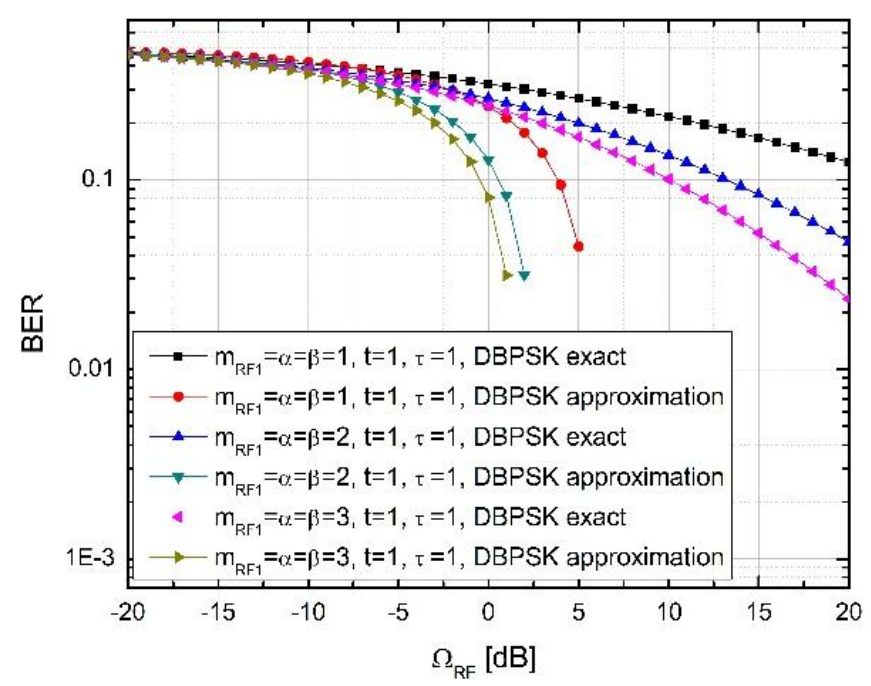

Figure 3. Comparison of exact and approximated results for BER versus $\Omega_{R F}$ under weak, moderate and strong TIF and nTIF channel conditions. 


\section{CONCLUSION}

This paper addresses the first -order outage statistics of the RF-FSO AFR dual-hop relay link. In particular, we provide closed form analytical expressions for $i$.) cumulative distribution function, ii.) outage probability and iii.) average bit error rate of the product of $\mathrm{Nm}$ and d-sNmRPs. Moreover, we provide comparison of novel exact analytical and LAM approximated closed form expressions under weak, strong and moderate nTIF and TIF channel conditions. Numerical examples show that exponential LAM approximations fit well with exact expressions for cumulative distribution function especially for higher output threshold $\mathrm{dB}$ values. On the other hand, exponential LAM fails to perform well in the case of $B E R_{R F-F S O}$, especially in higher RF average power $\mathrm{dB}$ regime. In general, nTIF and TIF severity parameters have more dominant impact on first order statistics then other observed parameters such as: type of binary modulation in case of $B E R_{R F-F S O}$. Our future works are going to include cooperative RF-FSO AFR relay systems.

\section{ACKNOWLEGMENTS}

The authors would like to acknowledge the COST Action 15104.This work has been partially funded from the VIU project of Tomsk Polytechnic University, Russia, No. 216/2019.

\section{REFERENCES}

Al-Ahmadi, S. 2014. The Gamma-Gamma Signal Fading Model: A Survey [Wireless Corner]. IEEE Antennas and Propagation Magazine, 56(5), $245-260$. doi:10.1109/map.2014.6971962

Andrews, L. C., \& Phillips, R. L. 2005. Laser Beam Propagation through Random Media. Washington: SPIE-Intl Soc Optical Eng. doi:10.1117/3.626196

Anees, S., \& Bhatnagar, M. R. 2015. Performance of an Amplify-and-Forward Dual-Hop Asymmetric RF-FSO Communication System. Journal of Optical Communications and Networking, 7(2), p. 124. doi:10.1364/jocn.7.000124

Butler, R. W., \& Wood, A. T. A. 2002. Laplace approximations for hypergeometric functions with matrix argument. The Annals of Statistics, 30(4), pp. 1155-1177. doi:10.1214/aos/1031689021

Douik, A., Dahrouj, H., Al-Naffouri, T. Y., \& Alouini, M. 2016. Hybrid Radio/Free-Space Optical Design for Next Generation Backhaul Systems. IEEE Transactions on Communications, 64(6), pp. 2563-2577. doi:10.1109/tcomm.2016.2557789

Gradshteyn, I. S., \& Ryzhik, I. M. 2010. Table of Integrals, Series, and Products. New York: Academic. 6th ed.

Hajri, N., Youssef, N., Kawabata, T., Patzold, M., \& Dahech, W. 2018. Statistical Properties of Double Hoyt Fading With Applications to the Performance Analysis of Wireless Communication Systems. IEEE Access, 6, pp. 19597-19609. doi:10.1109/access.2018.2820746

Hamza, A. S., Deogun, J. S., \& Alexander, D. R. 2019. Classification Framework for Free Space Optical
Communication Links and Systems. IEEE Communications Surveys and Tutorials, 21(2), pp. 1346-1382. doi:10.1109/comst.2018.2876805

Harding, M. C., \& Hausman, J. 2007. Using a Laplace approximation to estimate the random coefficients logit model by nonlinear least squares. International Economic Review, 48(4), pp. 1311-1328. doi:10.1111/j.14682354.2007.00463.x

Illi, E., Bouanani, F. E., \& Ayoub, F. 2017. A performance study of a hybrid 5G RF/FSO transmission system. In 2017 International Conference on Wireless Networks and Mobile Communications (WINCOM). Institute of Electrical and Electronics Engineers (IEEE)., pp. 1-7. doi:10.1109/wincom.2017.8238167

Issaid, C. B., \& Alouini, M. 2019. Level Crossing Rate and Average Outage Duration of Free Space Optical Links. IEEE Transactions on Communications, 67(9), pp. 6234-6242. doi:10.1109/tcomm.2019.2918324

Karimi, M., \& Masoumeh, N. 2011. Free space optical communications via optical amplify-and-forward relaying. Jour. of Light. Technology, 29(2), pp. 242-248.

Khalighi, M. A., \& Uysal, M. 2014. Survey on Free Space Optical Communication: A Communication Theory Perspective. IEEE Communications Surveys and Tutorials, 16(4), pp. 2231-2258. doi:10.1109/comst.2014.2329501

Milosevic, N., Stefanovic, M., Nikolic, Z., Spalevic, P., \& Stefanovic, C. 2018. Performance Analysis of InterferenceLimited Mobile-to-Mobile $\kappa-\mu$ Fading Channel. Wireless Personal Communications, 101(3), pp. 1685-1701.

Nakagami, M. 1960. The m-distribution-A general formula of intensity distribution of rapid fading. In W. G. Hoffman Ed., Statistical Methods in Radio Wave Propagation. Oxford: Pergamon.

Petkovic, M., Djordjevic, G. T., \& Djordjevic, I. B. 2017. Analysis of mixed RF/FSO system with imperfect CSI estimation. In 2017 19th International Conference on Transparent Optical Networks (ICTON). Institute of Electrical and Electronics Engineers (IEEE)., pp. 1-7. doi:10.1109/icton.2017.8024759

Petkovic, M. I., \& Trpovski, Z. 2018. Exact Outage Probability Analysis of the Mixed RF/FSO System With Variable-Gain Relays. IEEE Photonics Journal, 10(6), pp. 1-14. doi:10.1109/jphot.2018.2882611

Simon, M. K., \& Alouini, M. 2000. Digital Communication Over Fading Channels. New York, USA: Wiley. doi:10.1002/0471200697

Stefanovic, C. 2017. LCR of amplify and forward wireless relay systems in general alpha-Mu fading environment. In Proceedings 25th Telecommunication Forum. pp. 1-6.

Stefanovic, C., Pratesi, M., \& Santucci, F. 2018. Performance evaluation of cooperative communications over fading channels in vehicular networks. In Proceedings 2nd URSI Atalntic Science Radio Meeting (AT-RASC). pp. 1-4.

Stefanovic, D., Stefanovic, C., Djosic, D., Milic, D., Rancic, D., \& Stefanovic, M. 2019. LCR of the Ratio of the Product of Two Squared Nakagami-m Random Processes and Its Application to Wireless Communication Systems. In 2019 18th International Symposium INFOTEH-JAHORINA (INFOTEH).Institute of Electrical and Electronics Engineers (IEEE)., pp. 1-4. doi:10.1109/infoteh.2019.8717765 
Stefanovic, C., Pratesi, M., \& Santucci, F. 2019. Second Order Statistics of Mixed RF-FSO Relay Systems and its Application to Vehicular Networks. In Proceedings 2019 IEEE International Conference on Communications (ICC). pp. 1-6.

Talha, B., \& Pätzold, M. 2007. On the statistical properties of double Rice channels. In Proceedings 10th International Symposium on Wireless Personal Multimedia Communications, WPMC. pp. 517-522.

Vetelino, F. S., Young, C., Andrews, L., \& Recolons, J. 2007. Aperture averaging effects on the probability density of irradiance fluctuations in moderate-to-strong turbulence. Applied Optics, 46(11), p. 2099. doi:10.1364/ao.46.002099

Wang, J. 2010. Dirichlet Processes in Nonlinear Mixed Effects Models. Communications in Statistics - Simulation and Computation, 39(3),

pp.

539-556.
Zedini, E., Ansari, I. S., \& Alouini, M. 2015. Performance Analysis of Mixed Nakagami- \$m\$ and Gamma-Gamma Dual-Hop FSO Transmission Systems. IEEE Photonics Journal, 7(1), pp. 1-20. doi:10.1109/jphot.2014.2381657

Zedini, E., Ansari, I. S., \& Alouini, M. 2015. Unified performance analysis of mixed line of sight RF-FSO fixed gain dual-hop transmission systems. In 2015 IEEE Wireless Communications and Networking Conference (WCNC). Institute of Electrical and Electronics Engineers (IEEE), pp. 46-51. doi:10.1109/wcnc.2015.7127443

Zlatanov, N., Hadzi-Velkov, Z., \& Karagiannidis, G. 2008. Level crossing rate and average fade duration of the double Nakagami-m random process and application in MIMO keyhole fading channels. IEEE Communications Letters, 12(11), pp. 822-824. doi:10.1109/lcomm.2008.081058

doi:10.1080/03610910903511745 